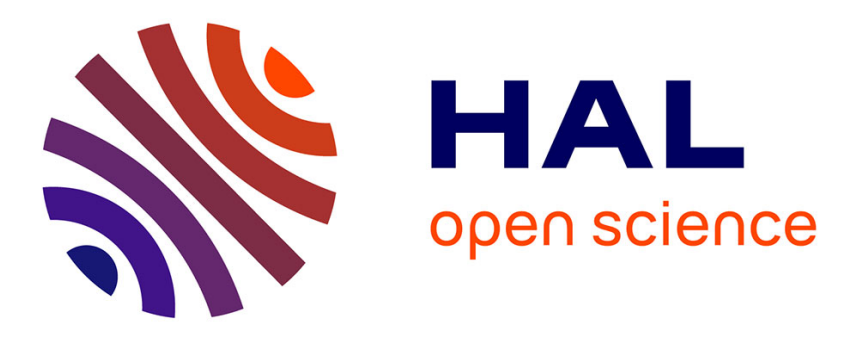

\title{
Représentations d'attachement chez les enfants, âgés de 3 à 7 ans, exposés aux violences conjugales
}

Laurence Berdot-Talmier, Christine Aubrion, Blaise Pierrehumbert, Chantal Zaouche Gaudron

\section{- To cite this version:}

Laurence Berdot-Talmier, Christine Aubrion, Blaise Pierrehumbert, Chantal Zaouche Gaudron. Représentations d'attachement chez les enfants, âgés de 3 à 7 ans, exposés aux violences conjugales. Devenir - Revue européenne du développement de l'enfant, 2016, 28 (1), pp.21-42. 10.3917/dev.161.0021 . hal-02150195

\section{HAL Id: hal-02150195 \\ https://hal-univ-tlse2.archives-ouvertes.fr/hal-02150195}

Submitted on 7 Jun 2019

HAL is a multi-disciplinary open access archive for the deposit and dissemination of scientific research documents, whether they are published or not. The documents may come from teaching and research institutions in France or abroad, or from public or private research centers.
L'archive ouverte pluridisciplinaire HAL, est destinée au dépôt et à la diffusion de documents scientifiques de niveau recherche, publiés ou non, émanant des établissements d'enseignement et de recherche français ou étrangers, des laboratoires publics ou privés. 


\title{
Représentations d'attachement chez les enfants, âgés de 3 à 7 ans, exposés aux violences conjugales
}

\author{
Laurence Berdot-Talmier'1, Christine Aubrion', \\ Blaise Pierrehumbert ${ }^{2}$ et Chantal Zaouche Gaudron ${ }^{1}$
}

${ }^{1}$ Université Toulouse - Jean Jaurès, UMR 5193 LISST-CERS

${ }^{2}$ Unité de recherche SUPEA, CHUV, Lausanne, Suisse

Remerciements : Les auteurs remercient les mères et les enfants qui ont accepté de participer à cette étude. Ils remercient également les professionnels du Centre d'Hébergement et de Réinsertion Sociale (C.H.R. S.) Olympe de Gouges à Toulouse qui leur ont permis de rentrer en contact avec ces familles.

Financements : Aucun

Conflits d'intérêts : Aucun 


\title{
Résumé
}

L'objectif de cette étude est d'analyser les représentations d'attachement auprès d'un échantillon clinique constitué de 10 enfants vivant en CHRS avec leur mère ayant été exposés aux violences conjugales. Au plan méthodologique, nous avons adapté le protocole de l'A.S.C.T (Bretherton, Ridgeway \& Cassidy, 1990) afin d'explorer les modèles internes opérants de ces enfants. Une grille d'observation a été établie afin de palier à l'absence de l'enregistrement vidéo habituellement préconisé. Au sein de notre échantillon clinique, les résultats obtenus mettent en évidence l'absence de représentation d'attachement qualifiée de « sécure ». Nos résultats semblent confirmer l'intérêt d'une telle évaluation clinique.

Mots clés : Relation d'attachement - Enfants exposés - Violence conjugale.

\section{Attachment representations in children exposed to domestic violence, aged 3 to 7 years old}

\begin{abstract}
The aim of this study is to analyze the attachment representations of a clinical sample of 10 children who stay with their mother in an Accommodation and Social Reintegration Centre and who have been exposed to domestic violence. As for methodology, the Attachment Story Completion Task (Bretherton, Ridgeway \& Cassidy, 1990) has been adapted in order to explore the internal operational models of these children. An observation grid has been developed to compensate for the absence of a video recording usually recommended. In our sample, the absence of the "secure" attachment representation is highlighted. Our results confirm the interest of this type of clinical evaluation.
\end{abstract}

Key words: Attachment-Children exposure- Domestic Violence 


\section{Introduction}

Les actions destinées aux enfants exposés à la violence conjugale ont émergé OutreAtlantique, et en particulier au Québec, voilà une trentaine d'années. En France, une femme meurt tous les deux jours et demi en moyenne, victime des violences de son compagnon (Ministère des Droits des Femmes, 2014). Dans près de 70\% des cas de violences conjugales, les actes se déroulent devant les enfants, et ils les concernent dans $10 \%$ des cas avec des séquelles physiques et psychologiques comparables à celles qui sont observées chez leur mère. Selon Sadlier (2010), les évènements les plus néfastes pour un enfant sont ceux qui se déroulent dans son premier milieu de vie, à savoir sa famille. Dans un tel contexte, le danger est d'autant plus grand que les acteurs - agresseur et victime -, ainsi que le décor dans lequel se déroule la scène sont familiers et constituent des références fondamentales pour l'enfant. Paul et Zaouche Gaudron (2013) considèrent la violence conjugale comme l'une des formes les plus insidieuses de maltraitance chez l'enfant. Pourtant cet objet de recherche reste encore très peu étudié en psychologie du développement.

\section{Cadre socio-politique et législatif}

L'Observatoire National de l'Enfance en Danger (2009) questionne la violence conjugale et la définit comme «des situations ou des faits de violence à la fois récurrents, souvent cumulatifs, s'aggravant et s'accélérant (phénomène dit de spirale) qui sont inscrits dans un rapport de force asymétrique (dominant / dominé) et figé ». La violence conjugale existe dans tous les pays, dans tous les groupes sociaux, économiques, religieux et culturels. Que ce soit en Amérique, en Europe ou au Québec, les femmes représentent 85 à 95\% des victimes. En France, l'Enquête Nationale sur les Violences Envers les Femmes, dite ENVEFF en 2000 (Jaspard, Brown, Condon, Fougeyrollas-Schwebel, Houel, Lhomond, Maillochon, SaurelCubizolles \& Schiltz, 2003), est la première enquête sur cette thématique. Plus récemment, celle intitulée «Cadre de vie et sécurité», fruit d'un partenariat entre l'INSEE et 
l'Observatoire National de la Délinquance et des Réponses Pénales (2012), a permis d'interroger, chaque année, depuis 2008, 13400 personnes entre 18 à 75 ans sur les actes de violences physiques ou sexuelles subis au cours des deux années précédant l'enquête et perpétrés par un conjoint ou ex-conjoint. Sur deux ans, les données chiffrées donnent une estimation de 404000 femmes « victimes déclarées » en France. La violence conjugale induit un important problème de santé publique dont on ne perçoit que la partie émergée qui amène les femmes à sortir du silence. Effectivement, les aspects les plus visibles sont les conséquences de la violence physique, alors que ce qui constitue également la violence, c'est bien le mode de relation des deux conjoints fondé sur la relation d'emprise, le contrôle et la domination d'un partenaire (le plus souvent le conjoint) sur l'autre.

La violence perpétrée directement sur les enfants est un problème reconnu par les états membres de 1'Union Européenne et par la Communauté Internationale depuis plusieurs années. C'est seulement en 2009 qu'une véritable reconnaissance des effets négatifs chez les enfants exposés à la violence conjugale voit le jour en France, grâce à l'avis du Comité Economique et Social Européen (COM, 2006).

\section{La violence conjugale}

Dans la violence conjugale, les rapports sont totalement asymétriques au sein du couple. Cette violence se distingue par quatre critères qui sont :

- Le pouvoir et l'emprise de l'un des partenaires sur l'autre conjoint.

- La volonté de domination et de contrôle de ce même partenaire.

- La persistance et la progression de ces violences dans le temps.

- L'impact sur la victime, ces violences suscitant peur, honte et baisse de l'estime de soi.

La violence conjugale est donc un système de relation dans lequel l'un des deux conjoints utilise la peur, l'intimidation, l'humiliation, les coups, le contrôle du temps ou des ressources 
financières. Comme l'explique Fortin, Coté, Rousseau et Dubé (2007), le cycle de la violence conjugale comprend quatre phases : la première phase correspond chez l'auteur à l'explosion de la violence résultant de l'accumulation de tensions ; s'ensuit la phase de «justification », de « réconciliation » durant laquelle l'auteur tente de se faire pardonner mais l'attribution de la responsabilité est toujours donnée à la victime afin de normaliser l'inacceptable ; enfin, une période de «lune de miel» durant laquelle l'auteur se montre affectueux, attitude lui permettant de garder le contrôle. Au fur et à mesure, les actes de violence augmentent en fréquence et en intensité. Pour Sadlier (2010), la violence conjugale se manifeste toujours dans les débuts par de la violence psychologique pernicieuse, difficile à reconnaître, tant par la victime que par l'auteur ou l'observateur extérieur. La relation d'emprise s'installe, la victime est persuadée de sa culpabilité, la violence quotidienne est devenue quasi ordinaire, entrainant une augmentation du seuil de tolérance de la victime. Selon Welzer-Lang (2005), la violence conjugale s'enracine dans les rapports sociaux de sexe. La violence masculine domestique est à mettre en rapport avec la domination/soumission dans les relations hommes/femmes. Le garçon ne naît pas violent, cette violence se construit socialement, tout comme la «condition» de «femme battue». Comme le soulignent Zaouche Gaudron et Molinier $(2012,122)$, « le passage à l'acte violent serait une protection pour lutter contre une dépendance excessive liées aux expériences maternelles, vulnérable au rejet, à l'abandon, à l'humiliation, en raison de ses vécus archaïques ». Selon Sadlier (2010), la violence conjugale n'est pas restreinte au couple conjugal bien au contraire, elle impacte toute la famille d'où les termes de « violence intrafamiliale » puis d' « enfant exposé » à cette violence.

\section{L'enfant exposé aux violences conjugales}

L'exposition de l'enfant à la violence conjugale concerne tout ce que l'enfant voit, entend et perçoit au cours de l'épisode violent. En France, selon l'estimation nationale solidarité femmes en 2006, à partir des chiffres de l'ENVEFF, dernier recensement en date (INED, 
2014) le nombre d'enfants aux prises avec cette problématique s'élèverait à 4 millions. La première difficulté dans cette problématique réfère au caractère caché de la violence conjugale comme d'ailleurs dans les situations de maltraitance. En effet, dans ces situations, la « loi du silence » est de mise, à l'intérieur même de la famille tout comme à l'extérieur du cercle familial. Toutefois, si on interroge directement les enfants vivant dans ce contexte de violence au sein du couple, on constate qu'ils mentionnent presque tous y avoir été exposés.

L'exposition à la violence conjugale est une expérience complexe qui peut être vécue de différentes façons par l'enfant. Tout d'abord, l'enfant pressent souvent que dévoiler les violences qu'il rencontre dans la sphère familiale risque d'ébranler les fondements familiaux et cela l'oblige à vivre dans le secret, l'enfermant de ce fait dans la culpabilité (Sadlier, 2010). Certains enfants peuvent s'identifier à l'agresseur, d'autres se sentent responsables de cette violence et ont tendance à se blâmer ce qui entraine honte et culpabilité (Sadlier, 2010). Le déni de cette violence peut être un des moyens de défense utilisé par l'enfant, le protégeant un temps d'autres situations stressantes. Un conflit de loyauté va survenir chez l'enfant, lorsqu'il se sent dans l'obligation de prendre position par rapport à l'un ou l'autre de ses parents. Effectivement, vivre dans un climat de violence conjugale va signifier pour l'enfant, outre toute la terreur que celle-ci va provoquer chez lui, une détresse liée à l'importance des conflits de loyauté et à l'ambivalence que l'enfant va éprouver. Il va se retrouver tiraillé entre l'amour qu'il ressent pour ses deux parents et le fait qu'aimer le parent agresseur pourrait être appréhendé comme une trahison du point de vue du parent qui en est victime. L'enfant vit dans un « environnement toxique ». La violence dont il est témoin a les mêmes effets sur lui que s'il était directement victime de violence physique. L'indisponibilité psychique et physique de la mère, elle-même en grande souffrance, peut perturber le développement de l'enfant qui devient ainsi victime de violences psychologiques. Il se retrouve dans une position de détresse intense et son comportement va se désorganiser (Savard \& Zaouche 
Gaudron, 2011). L'état de stress post-traumatique (Savard \& Zaouche Gaudron, 2010, 2011, 2013) est la plupart du temps observé chez les enfants exposés à la violence conjugale, en particulier chez les plus jeunes. En effet, comme le souligne la revue de littérature, chez ces derniers, la probabilité de retrouver des troubles psychopathologique est plus élevée (Guédeney, Guédeney \& Rabouam, 2013). La pathologie post-traumatique est constituée par trois grandes catégories de symptômes : le syndrome de répétition, l'évitement ou l'inhibition et l'hyperactivité neurovégétative. Sur les plans affectif et émotionnel, les résultats indiquent que, dans un contexte de violence conjugale, les enfants exposés ont une santé émotionnelle beaucoup plus fragile et vont manifester beaucoup plus de troubles intériorisés que les enfants de mères non violentées. À ces troubles intériorisés tels que la dépression, l'anxiété, une faible estime de soi, une construction identitaire fragilisée, vont s'ajouter de nombreux troubles extériorisés, comme l'agressivité ou l'hyperactivité. Toutefois, il est important de préciser que les enfants ne vont pas tous être affectés de la même manière et avec la même intensité. Effectivement, le jeune âge constitue un facteur de risque supplémentaire de par une grande dépendance dans la satisfaction des besoins physiques, psychiques et émotionnels de l'enfant envers ses parents (Sadlier, 2010). Concernant la manière dont les mères interagissent avec leur enfant, les recherches donnent des résultats hétérogènes. Bien qu'étant plus stressées, certaines d'entre elles adoptent des stratégies comparables à celles des mères non violentées, avec parfois, une empathie plus importante envers leur enfant afin de le protéger et le soutenir par rapport à ce qu'ils vivent. D'autres études font état de moins de disponibilité émotionnelle envers l'enfant et deux fois plus de violences physiques pour «l'éduquer». La référence à la disponibilité émotionnelle amène à considérer le modèle théorique de l'attachement (Prior \& Glaser, 2010). En effet, le développement de tout être humain étant en partie façonné par les expériences vécues durant l'enfance, il est important de déterminer les facteurs liés à l'exposition à la violence conjugale qui auront des répercussions potentielles 
sur la personnalité, le comportement et les attitudes de l'enfant voire de l'adulte qu'il deviendra.

\section{La théorie de l'attachement et les enfants exposés à la violence conjugale}

L'attachement est un processus de nature émotionnelle organisé par des affects particuliers éprouvés par le sujet en interaction, résultant d'une constante adaptation entre l'enfant et le ou les figures d'attachement. Les parents qui interagissent de façon prévisible, cohérente et chaleureuse avec leur enfant et ce, dès la naissance permettent la construction d'un lien sécure. Le caregiver sécure se trouve être un «port d'attache » pour l'enfant qui va alors pouvoir accepter la séparation lui permettant d'explorer l'environnement. Bowlby (1973) va mobiliser le concept de Modèle Interne Opérant (M.I.O) afin de désigner les processus mentaux qui vont se construire à partir des expériences relationnelles d'attachement. Le rôle des M.I.O est de fournir une représentation mentale du monde pour aider l'individu à percevoir et à interpréter les évènements, à les anticiper afin de planifier en retour son comportement (Guédeney, N., 2010). Cette représentation mentale va donc se construire au fur et à mesure des interactions : elle s'établit entre 6 et 9 mois et va se stabiliser autour des 56 ans mais, dès l'âge de 2-3 ans, l'enfant commence à avoir une représentation mentale sur ses relations d'attachement (Prior \& Glaser, 2010). Ces modèles imposent également une «grille de lecture » personnelle des évènements vécus qui sera réactivée lors de situations contextuelles telles que la détresse, la menace ou le danger en relation avec le système d'attachement. Selon N. Guédeney (2010), ces modèles se construisent progressivement en fonction des réactions des figures d'attachement aux besoins du jeune enfant pour s'inscrire alors au niveau des représentations plus ou moins conscientes de ce dernier. Lorsque les besoins d'attachement de l'enfant n'obtiennent pas de réponses adéquates, l'enfant construit progressivement des stratégies ayant différentes modalités. Ce sont des stratégies que l'on rencontre chez les enfants dont le mode de représentation d'attachement est qualifié 
d'organisé mais « insécure » (Prior \& Glaser, 2010). Par contre, si l'environnement répond de manière « terrifiante » pour l'enfant, ces stratégies ne sont d'aucun secours, c'est ce que l'on remarque chez l'enfant dont le mode de représentation d'attachement est désorganisé (op.cit.). La régulation des émotions est donc un processus central dans la compréhension des différentes stratégies mobilisées (Savard \& Zaouche Gaudron, 2011). Lyons-Ruth et Jacobvitz (1999) soulignent que la prise en compte de la peur chez l'enfant est essentielle à la conception de la notion de désorganisation. Le caregiver de ces enfants serait à la fois source de réconfort et d'inquiétude. Lyons-Ruth (2007) élargit le cadre de référence en postulant que l'absence de régulation affective, provenant du donneur de soins, peut entrainer la désorganisation de l'enfant plus que la crainte du parent en soi.

Dans la méta-analyse effectuée par IJzendoorn, Schuengel et Bakermans-Kranenburg (1999), $15 \%$ de nourrissons «tout venant» manifestent aussi un attachement désorganisé. Des comportements moins extrêmes de la part du caregiver peuvent donc aussi être à l'origine d'un lien d'attachement désorganisé. La désorganisation est donc largement associée aux processus perturbés de la communication affective entre l'enfant et le donneur de soins. De plus, il est intéressant de noter que dans une population très stressée et de bas revenu, on repère chez les enfants, dont les mères peuvent établir des configurations de communication affective non perturbée, un taux très bas de désorganisation.

Notre recherche s'inscrit dans la lignée des travaux nord-américains (Lyons-Ruth, 2007) et dans ceux de Savard et Zaouche Gaudron (2011), de Zaouche Gaudron et Paul (2014), en France. Les résultats de Savard et Zaouche Gaudron (2011), dans une étude portant sur l'exposition à la violence conjugale, vont dans le sens de ceux obtenus par Zeanah, Danis, Hirshberg, Benoit, Miller et Heller en 1999, à savoir : seulement $36 \%$ des enfants développent une représentation d'attachement de type sécure, alors que $47 \%$ d'entre eux développent une représentation d'attachement de type désorganisé. Zeanah et al. (1999) qui 
ont travaillé auprès d'enfants âgés de 15 mois, exposés à la violence conjugale, ont trouvé que $56,9 \%$ présentaient un attachement désorganisé, contre uniquement 37,5\% d'attachement de type sécure. Cette répartition diffère grandement de la distribution normale des profils d'attachement qui se retrouve dans la méta-analyse effectuée en Amérique du Nord par IJzendoorn et al. (1999), chez des enfants tout venant, puisque $62 \%$ des enfants sont classés comme sécure et seulement $15 \%$ comme désorganisés.

Lyons-Ruth et Jacobvitz (1999) soulignent que la notion de peur dans la théorie de l'attachement est essentielle à la compréhension de la désorganisation. Or, les soins fournis dans le contexte imprévisible et parfois chaotique des familles où règne de la violence conjugale ainsi que le cycle de cette dernière, peuvent conduire à une mauvaise régulation des besoins d'attachement de l'enfant. Comme le soulignent IJzendoorn et BakermansKranenburg (2010), lorsque les personnes qui doivent prendre soins de l'enfant négligent le temps et la qualité d'interaction avec lui, pour cause de tracas ou de problèmes urgents dans d'autres sphères de leur quotidien, il se crée alors un système d'attachement hypervigilant, ou diffus chez l'enfant, qui ne sait vers qui se tourner pour trouver du réconfort dans les moments de tension.

Les résultats des études (IJzendoorn \& al., 1999, Miljkovitch, Pierrehumbert, Karmaniola \& Halfon, 2003) révèlent que les M.I.O de l'enfant peuvent s'avérer une voie fructueuse dans le champ de la prévention. Selon Miljkovitch et al. (2003), le type de M.I.O de l'enfant renseigne sur sa capacité ou son impuissance à surmonter les épreuves. Ces éléments confirment l'importance de prendre en compte la désorganisation des stratégies d'attachement des enfants pouvant entrainer des difficultés dans leurs relations affectives futures, dans la confiance en eux-mêmes et aux autres tout au long de leur vie. 
Par conséquent, l'objectif de cette étude est d'examiner quels types de représentations d'attachement développent les enfants entre 3 et 7 ans, ayant été exposés au contexte de violence conjugale.

\section{Méthode}

\section{Participants et procédure}

L'échantillon de cette étude est composé de 10 enfants, dont 2 filles et 8 garçons, âgés de 3 à 7 ans $(M=4,9 ; \sigma=1,72)$. Seules les deux filles sont des enfants uniques. Les huit garçons font tous partie d'une fratrie de deux enfants, tous deux ayant participé au protocole. Ces dix enfants ont été rencontrés dans un Centre d'Hébergement et de Réinsertion Sociale (C.H.R.S.) de la région toulousaine, accueillant les femmes victimes de violences conjugales accompagnées de leurs enfants, au cours des 6 mois d’hébergement consentis par la loi. Ces mères sont toutes séparées de leurs conjoints. Elles sont âgées de 21 à 30 ans $(\mathrm{N}=6 ; \mathrm{M}=$ 27,5; $\sigma=3,39)$. L'une d'entre elles occupe un emploi en tant que personnel de service alors que toutes les autres sont sans emploi. Elles sont toutes originaires du continent Africain.

Nous avons présenté la recherche à ces mères au cours d'un groupe de parole au sein du C.H.R.S. en stipulant que le thème des violences conjugales ne serait pas abordé avec l'enfant. Les mères ont donné leur accord par écrit afin que leurs enfants participent à notre recherche. Le comité d'éthique n'a pas été consulté mais un accord de recherche distribué à ces mères rappelait le code de déontologie et d'éthique de la recherche en psychologie. Nous avons également demandé à l'enfant s'il consentait à participer à notre travail. Le formulaire de consentement expliquait le but de la recherche, tout en garantissant l'anonymat et la confidentialité des données. Au regard de la problématique des enfants rencontrés, il ne nous a pas été possible d'utiliser le support vidéo normalement préconisé dans le protocole utilisé. 
Nous avons donc réalisé une passation en binôme avec un enregistrement audio et une cotation « papier-crayon ».

Chaque enfant a été reçu individuellement, durant une trentaine de minutes, dans une salle mise à disposition au sein du C.H.R.S durant les temps d'activités en groupe, proposés par l'éducatrice de jeunes enfants au sein de la résidence. Pendant que l'une des expérimentatrices faisait passer le protocole à l'enfant, l'autre observait et renseignait la grille de cotation construite à cet effet $(C f$. Tableau 1). Après la passation du protocole par chaque enfant, nous prenions le temps nécessaire à la cotation du C.C.H, en confrontant à la fois l'enregistrement audio à la grille de cotation dûment remplie.

\section{Présentation de l'outil et procédure}

Il existe différents outils permettant d'appréhender la qualité des relations et des représentations d'attachement du jeune enfant (Prior \& Glaser, 2010). Bien souvent, dans les méthodologies utilisées, les auteurs indiquent que les échantillons sont constitués de dyades mères-enfants, l'enfant n'est pas systématiquement interrogé et les études se basent fréquemment sur les dires et les représentations de la mère à propos de l'enfant. Il existe pourtant des outils permettant d'évaluer et d'interroger directement l'enfant, sans pour autant le confronter à des situations anxiogènes. En 2008, Zaouche Gaudron et Pierrehumbert ont réalisé un état de la question concernant les travaux menés en France et à l'étranger sur l'application de ce modèle dans des circonstances diverses. Notre choix s'est porté sur le protocole «Attachment Story Completion Task» ou A.S.C.T, élaboré par Bretherton, Ridgeway et Cassidy (1990), qui nous permet d'examiner directement les représentations de l'enfant et non celles de son entourage. Il permet notamment d'explorer les Modèles Internes Opérants de l'enfant d'âge pré-scolaire. Effectivement, à partir de la période préscolaire, le langage et les habiletés représentationnelles évoluent, et l'accès aux M.I.O devient possible par des techniques telles que la narration d'histoires avec la manipulation de figurines. 
Dans ce protocole, six amorces d'histoires sont présentées successivement à l'enfant. Ces histoires mettent en scène des figurines représentant les membres d'une même famille (père, mère, grand-mère et deux enfants du même sexe que l'enfant interrogé). Il est ensuite demandé à l'enfant de compléter les histoires, en manipulant les figurines. La mise en scène de ces histoires va activer son système d'attachement, afin qu'il établisse les scripts des relations d'attachement contenu dans ses M.I.O. Chaque petite histoire va demander un matériel spécifique, en fonction de la consigne de départ. La passation doit se dérouler dans une position de face à face avec l'enfant, et il est préférable d'utiliser du mobilier spécifique pour enfant (petite table), afin que celui-ci puisse manipuler les figurines à son aise, ce que nous avons fait.

- La première histoire (le gâteau d'anniversaire) ne rentre pas dans le système de codage. Elle va permettre à la fois de rentrer en contact avec l'enfant et de lui faire comprendre ce qui est attendu de sa participation, tout en lui permettant de se familiariser avec le matériel.

- La deuxième histoire est celle du jus de fruit renversé par l'enfant. Cette histoire a pour objectif de mettre en lumière la façon dont l'enfant va mettre en scène le comportement de réprimande de son père et/ou de sa mère, ainsi que ses réactions face à ce comportement.

- La troisième histoire est la sortie dans le parc et la blessure au genou. L'intention sera ici de voir comment l'enfant va réagir face à sa souffrance et surtout d'observer comment il imagine le comportement de ses parents, face à sa blessure et à son chagrin.

- La quatrième histoire est celle du monstre dans la chambre. La visée est ici, de voir comment l'enfant perçoit le soutien de ses parents lorsqu'il est anxieux et angoissé.

- Les deux dernières histoires mettent en scène la problématique de la séparation ce qui va être susceptible d'engendre chez l'enfant des émotions particulières en lien avec la séparation : angoisse de séparation, peur de l'abandon. L'objectif est d'analyser les craintes 
que peut avoir l'enfant face à cette séparation annoncée et d'observer la perception qu'a l'enfant du comportement parental lors de la séparation.

- La sixième et dernière histoire est celle des retrouvailles. Le but est ici aussi d'analyser les émotions de l'enfant face aux retrouvailles et la façon dont il va se représenter le comportement de ses parents. A la fin du protocole, une phase de jeu libre est proposée à l'enfant et tous les jouets utilisés précédemment sont remis à sa disposition afin de donner à l'enfant un moment d'activité ludique, libre de toute contrainte.

\section{Tableau 1}

Nous avons associé cet outil au système de cotation de Cartes pour Complément d'Histoires (C.C.H) (Miljkovitch \& al., 2003) qui permet de mesurer les stratégies d'attachement des enfants au niveau des représentations, tout en caractérisant leur façon de construire un narratif. Ce questionnaire, sous forme de Q-Sort, va permettre, à partir de 65 items présentés sur des cartes, le codage global des données observées. Le calcul des scores obtenus par l'enfant se fait sur la base d'un tri forcé de cartes permettant de coter le type de représentation ou M.I.O de l'enfant (securé, désactivé, hyperactivé ou désorganisé). En 2008, Miljkovitch et Pierrehumbert ont spécifié l'outil de codage grâce à la méthode des « seuils avec privilège pour la sécurité et la désorganisation » (Cf. Tableau 2$)$. Celle-ci réside dans la prise en compte de la moyenne et de l'écart-type des scores obtenus aux quatre types de stratégies, avant d'être à même de pouvoir se prononcer sur la qualité des représentations d'attachement de l'enfant. Pour pallier à l'absence du support vidéo, nous avons construit une grille d'observation afin de saisir l'attitude générale de l'enfant, son comportement envers les poupées adultes, son expressivité ainsi que son comportement et attitude lors des phases de séparations et de réunions présentées dans les scénarii des histoires. En complément du support audio, cette grille d'observation, élaborée à partir de certains des 65 items du C.C.H., nous a permis d'effectuer le tri de carte conformément à ce qu'avait pu manifester l'enfant 
durant la passation. Après avoir repris les grilles d'observations et l'enregistrement audio pour chaque enfant, les cartes sont donc triées par les expérimentatrices, selon un protocole qui va consister à classer chacune d'elles. Le regroupement des scores pour chaque item en 7 échelles va être obtenu, permettant de décrire certaines caractéristiques du jeu et du contenu narratif dont: collaboration, représentation de soutien parental, narratif positif, expression d'affects appropriée, réaction à la séparation, distance symbolique, faible compétence narrative (Miljkovitch \& al., 2003).

Tableau 2

\section{Résultats}

Dans notre échantillon $(\mathrm{N}=10)$, six enfants présentent une représentation d'attachement désorganisé et quatre enfants une représentation d'attachement désactivé. Nous n'avons retrouvé aucun enfant ayant une représentation d'attachement qualifiée de « sécure » $(C f$.

Tableau 3). Les résultats obtenus nous montrent que les quatre prototypes d'attachement décrits par Miljkovitch et al. (2003) ne sont pas présents au sein de notre échantillon, contrairement aux résultats trouvés dans la recherche de Savard (2011).

\section{Tableau 3}

De façon générale, lors de la passation de l'A.S.C.T, les six enfants dont les représentations d'attachement sont désorganisées se sont exprimés par des narratifs marqués par une perte de contrôle dans les situations proposées. L'anxiété étant trop importante à gérer, trois enfants sont restés totalement silencieux, ne montrant pas plus d'investissement dans le jeu scénique avec les figurines face aux histoires proposées que dans leur narratif. Deux enfants, quant à eux, faisaient émerger des attitudes de destruction, au cours desquelles aucun des personnages n'était en mesure de faire face à la situation. Pour l'un d'entre eux, on note des inversions de rôles entre les personnages parents/enfant avec une « parentification » de l'enfant qui a pu être 
observé. L'attitude la plus prégnante de ces enfants s'est traduite par un sentiment de trouble, de gêne, d'inconfort face aux histoires à compléter.

Les conduites des 4 enfants classés désactivés expriment une angoisse face à la construction d'un narratif verbal et/ou scénique lors des compléments d'histoires. Les enfants sont réticents à participer de façon impliquée et approfondie, se réfugiant derrière des stéréotypes de «bons parents » et « d'enfants modèles ». On note peu d'échanges avec les personnages, les rapports d'aides et de protections évoqués au cours de l'élaboration narrative et scénique restent plaqués et superficiels. Lors de la phase de retrouvailles, les personnages enfants se détournent de la scène afin de réprimer les affects correspondant à cette situation (Miljkovitch \& al., 2003). Les enfants de type désactivés utilisent comme mode de protection la mise à distance de leurs sentiments et de leur attention face à tout ce qui relève de l'attachement.

Concernant les résultats obtenus à partir des sept échelles, pour tous les enfants de notre échantillon, les habilités à collaborer, le soutien parental, les affects appropriés, la distance symbolique et les compétences narratives sont plutôt pauvres et fragiles. En effet, l'analyse détaillée de la répartition des scores T aux 7 échelles du C.C.H indique que sur l'échantillon général, les enfants rencontrent des problèmes à toutes les échelles du C.C.H. De façon plus précise, les résultats indiquent :

- L'échelle « collaboration » nous renseigne sur la volonté de l'enfant à se prêter au jeu et à y participer. Au vu des résultats, un seul enfant (enfant 7) a obtenu un chiffre légèrement supérieur à la moyenne $(56,38)$, les autres se situant tous en dessous $(M=35,62 ; \sigma=15,54)$. Selon Miljkovitch et al. (2003), ces résultats tendraient à montrer un refus ou une incapacité de la plupart de ces enfants à porter leur attention sur des questions d'attachement.

- L'échelle « représentation de soutien parental » renvoie à des contenus de représentations et aux modèles de relations que l'enfant a intériorisés à partir des interactions qu'il a pu avoir 
avec ses parents. Tous les enfants de notre échantillon ont un score inférieur à la moyenne, allant de 22,62 à 41,96 (M=35,22; $\sigma=8,81)$.

Ces faibles scores suggèrent que les enfants n'ont pas intégré un modèle de relation dans lequel la figure d'attachement est perçue comme sensible et sécurisante. Parfois même, c'est l'enfant lui-même qui endosse ces caractéristiques en présentant le personnage enfant comme sensible et sécurisant. On note ici que les scores diminuent proportionnellement avec l'âge des enfants. Plus l'enfant est jeune, plus son score est élevé, toutefois il reste en dessous de la moyenne. On peut émettre l'hypothèse que l'enfant le plus âgé de notre échantillon peut de par, entre autres, ses facultés cognitives, endosser des rôles parentaux (processus de « parentification ») et avoir une image moins idéalisée des figures parentales. A l'inverse, le plus jeune ayant encore besoin de l'étayage parental va, malgré les difficultés induites par la situation de violence conjugale, préserver une représentation positive de ses parents.

- L'échelle « narratif positif» mesure la tendance à construire des histoires sous un jour positif, les figures parentales sont présentées comme affectueuses et les enfants comme étant heureux. Tous les enfants de notre échantillon ont un score $\mathrm{T}$ inférieur à la moyenne, allant de 31,72 à 45,39 $(M=39,86 ; \sigma=5,84)$. La tonalité générale des récits est plutôt empreinte d'un sentiment positif affaibli. Dans les récits des enfants, les parents sont effectivement peu décrits comme ayant des conduites affectives envers l'enfant.

- Un faible score à l'échelle « expression d'affect appropriée » indique une tendance à exprimer les émotions d'une manière directe et inappropriée, comme par exemple un « passage à l'acte » sur le matériel. Les émotions sont peu exprimées par le biais symbolique du jeu. Tous les enfants ont des scores $\mathrm{T}$ à cette échelle inférieurs à la moyenne, allant de 26,85 à $43,36(\mathrm{M}=33,04 ; \sigma=7,34)$. On peut donc dire qu'ils ont une capacité réduite à évoquer et à contenir leurs affects. Au vu de la passation, il est possible d'inférer que les 
scènes d'attachement déstabilisent et mobilisent fortement les émotions des enfants de notre échantillon.

- L'échelle « réaction à la séparation » témoigne de l'aptitude de l'enfant à ne pas accepter le départ des parents et, des stratégies qu'il va mettre en place pour les retenir. Pour cette échelle, un faible score $\mathrm{T}$ correspond à un enfant étant en mesure de représenter les personnages comme acceptant la situation de séparation et y faisant face. Tous les enfants ont un score $\mathrm{T}$ à cette échelle inférieur à la moyenne, allant de 36,67 à 42,86 $(\mathrm{M}=40,27 ; \sigma=$ 2,59). Lors des passations, les enfants ont globalement accepté la situation de séparation proposée, parvenant à adopter des stratégies pour y faire face et ne cherchant pas à empêcher le départ.

- L'échelle « distance symbolique » renvoie au maintien d'une distance par rapport aux actes et aux émotions qui sont évoqués lors du jeu. Tous les enfants de notre échantillon ont un score $\mathrm{T}$ inférieur à la moyenne, allant de 32,01 à 44,02 $(\mathrm{M}=36,81 ; \sigma=5,18)$. Effectivement, lors des passations, l'enfant se distingue peu des personnages de l'histoire et les utilisent comme s'il était lui-même l'un des personnages. Les enfants ont des difficultés à maintenir de la distance par rapport aux actes et aux émotions évoquées lors du jeu qui leur est proposé.

- Enfin, l'échelle « faible compétence narrative » se manifeste par une impossibilité à produire des narratifs cohérents et structurés. Les enfants qui ont un faible score à cette échelle complètent les histoires avec facilité. Un seul des enfants (enfant 7) de notre échantillon obtient un score T pour cette échelle inférieure à la moyenne, soit 39,2. Les autres enfants se situent au-dessus de la moyenne, allant de 55,39 à 81,31 $(\mathrm{M}=57,82 ; \sigma=17,41)$. En règle générale, les enfants n'ont que près peu mobilisé les personnages dans les scénarii. Les compétences narratives s'accroissant avec l'âge des enfants, le plus jeune (enfant 4, 3 ans) présente des compétences narratives particulièrement faibles, a contrario l'enfant (enfant 7, 7 ans) l'un des plus âgé obtient des compétences narratives plus efficientes. De façon générale, 
les enfants éprouvent des difficultés à activer leur système d'attachement pour favoriser la proximité avec le parent lorsqu'ils se trouvent dans des situations alarmantes.

\section{Discussion}

L'objectif de cette recherche est donc d'examiner l'influence de l'exposition à la violence conjugale, au regard des représentations d'attachement développées par 10 enfants âgés de 3 à 7 ans, ayant vécu dans ce contexte familial. Concernant les résultats obtenus dans notre recherche, les enfants de notre échantillon possèdent tous une représentation d'attachement de type insécure, 4 enfants sur 10 sont classés comme désactivés et 6 enfants sur 10 sont classés comme désorganisés. Les narratifs des enfants sont souvent incohérents, marqués par une perte de contrôle de la situation, avec des personnages fréquemment représentés comme impuissants. Les enfants éprouvent des difficultés à faire intervenir les personnages incarnant les figures parentales, notamment le père. En effet, les résultats obtenus au score $\mathrm{T}$ correspondant aux 4 dimensions de représentation d'attachement de l'enfant, tout comme les scores T obtenus par les enfants de notre échantillon aux 7 échelles sont faibles. Au regard des résultats obtenus dans notre échantillon, nous pouvons supposer que ces enfants ont eu beaucoup de mal à se saisir et à résoudre les problèmes soulevés par les débuts d'histoires proposées. Nos résultats vont dans le sens des études d'Ijzendoorn et al. (2010) ne mettant en évidence que $14 \%$ de profils d'attachement sécurisés contrairement à la distribution normale des profils d'attachement chez des enfants tout venant (Ijzendoorn \& al., 1999 ; Zeanah \& al., 1999). Nous pouvons maintenant avancer l'hypothèse que l'exposition de l'enfant aux violences conjugales (et pas seulement victimes de violences parentales), constitue également un risque majeur de développer un attachement de type insécurisé. Savard (2011) précise également que la violence conjugale active fortement le système d'attachement de l'enfant. Il est alors confronté à la recherche d'une base de sécurité chez ses parents et à l'indisponibilité de ces derniers qui ne sont pas en mesure de rassurer l'enfant face à la violence qui imprègne 
le foyer parental. L'enfant se retrouve donc dans une situation qui induit des comportements d'approche/évitement, le maintenant dans une position d'effroi, sans possibilité de trouver de solutions adaptées. On comprend alors comment après avoir vécu à maintes reprises ce dilemme, l'enfant développe des représentations d'attachement de type désorganisées. La violence conjugale dont les enfants sont témoins suscite chez eux de l'anxiété au sujet de la capacité de leur mère à les protéger et à se protéger elle-même contre la violence. Sadlier (2010) note que dans un foyer où règne la violence conjugale, la chronicité de la violence peut empêcher la mère qui vit avec l'auteur de protéger son enfant de l'exposition aux évènements effrayants et anxiogènes. De ce fait, lors d'un attachement insécure, les enfants vont développer des conduites pathologiques d'autorégulation liées à leur propre détresse et peuvent, dans certains cas, chercher à co-réguler les états affectifs des parents. Pour certains auteurs, une posture d'enfant «parentalisé », tendrait à préserver le bien-être, soit de la victime, soit de l'auteur des violences ou des deux. Nous émettons l'hypothèse que la parentalisation entraîne une agonie psychique de l'enfant laissant peu de place à une structuration psychique satisfaisante. Comme le souligne Macfie, Fitzpatrick, Rivas et Cox (2008), l'inversion du rôle parental est souvent observée chez ces enfants exposés à la violence conjugale. Concernant notre étude, cette «parentification» se retrouve de façon prégnante dans les résultats obtenus par l'un des enfants. En effet, celui-ci a agi en tant que parent de sa fratrie et de la victime. Tout au long de la passation, cet enfant tient un rôle pacificateur, faisant en sorte de réguler, voire d'éliminer les conflits émergeants dans chaque début d'histoire. Dans leur recherche, Fortin et Lachance (2011) révèlent que la sévérité et la fréquence des violences ainsi que la détresse des mères et la qualité de la relation mère-enfant prédisent la «parentification » de l'enfant.

Chez les enfants 2 et 3 ayant respectivement 5 et 7 ans et des représentations d'attachement désorganisés, on note des comportements destructeurs, un jeu remettant en scène de manière 

répétitive les évènements traumatiques ou des thèmes qui y sont associés. En effet, lors de la passation, leur production scénique et leur narratif n'ont été qu'agressions, colère et destruction. Ce comportement pourrait laisser présager, chez ces enfants, une mise en avant de ce qu'ils ont vu et perçu au sein de leur foyer. Il est possible de relier cette mise en scène avec les données présentées par Sadlier (2010) qui révèle l'occurrence chez les enfants exposés aux violences conjugales d'un trouble post-traumatique. Vouche (2011) répertorie les symptômes pouvant être rencontrés chez ces enfants en fonction des différentes étapes de leur développement; chez les enfants d'âge préscolaire, ce sont des actes d'agression parfois de cruauté, de destruction de biens. Un tel comportement de la part de l'enfant peut être sanctionné, parfois sévèrement, sans une prise en compte des causes inhérentes à ce comportement, à savoir la violence vécue dans le foyer familial. Le problème sous-jacent étant de ce fait occulté, ces enfants ne reçoivent pas le type d'aide approprié. De plus, certaines caractéristiques vont constituer des facteurs de risque et/ou de protection. Sont considérés comme facteurs de risque : le jeune âge de l'enfant qui va affecter la perception que celui-ci possède de son parent et le fait d'être de sexe masculin. Les garçons exposés à la violence conjugale présentent plus de difficultés que les filles en ce qui concerne les problèmes extériorisés. Au vu de son jeune âge, le comportement de l'un des enfants (enfant 2) peut aussi être relié aux processus imitatifs. Les travaux sur l'imitation et les jeux de simulacre, sont intéressants pour illustrer cette constatation. L'imitation est un acte constitutif de l'identité chez l'enfant et les actes imitatifs ne se font qu'à partir des personnes qui exercent une profonde influence sur l'enfant (affective, autorité...). Ainsi, certains enfants peuvent «rejouer» la violence, car ils ont intégré que cette agressivité était une façon acceptable de résoudre les conflits.

\section{Conclusion}


En nous basant sur les points de vue des enfants exposés à la violence conjugale, cette recherche apporte des connaissances relatives à leurs représentations d'attachement qui, à ce jour, restent encore trop méconnues dans la littérature scientifique pour cette tranche d'âge. Concernant les résultats obtenus, les 10 enfants de notre échantillon possèdent tous une représentation d'attachement de type insécure, 4 enfants sont classés comme désactivés et 6 enfants sont classés comme désorganisés.

L'outil et la procédure utilisés semblent confirmer l'intérêt d'une évaluation sans support vidéo auprès d'une population clinique. Mais, les résultats que nous avons obtenus ne peuvent être généralisés à la population d'enfants exposés aux violences conjugales en raison de la taille restreinte de notre échantillon. De plus, ayant recueilli les informations à un seul moment de leur parcours de vie, nous ne pouvons préjuger de la stabilité de nos résultats au fil du temps.

Pour autant, certains éléments méritent d'être relevés. La détérioration de la relation d'attachement que nous avons précisée dans notre échantillon est extrêmement importante à prendre en compte dans la mesure où elle pourra par la suite être l'amorce d'autres effets délétères tout au long de sa vie, tels que des relations affectives futures détériorées, une estime de soi faible...Par ailleurs, il paraît tout aussi important de travailler avec l'enfant l'ambivalence ressentie envers l'auteur ou la victime. Sadlier (2010) souligne, en effet, l'intérêt pour certains objectifs thérapeutiques tels que la verbalisation de la violence vue, entendue ou vécue, l'expression et la gestion des émotions telles que la peur, la colère ou la honte afin de soutenir leur développement. L'exposition à la violence conjugale durant l'enfance représente un facteur de vulnérabilité contribuant à la victimisation à l'âge adulte et la transmission intergénérationnelle de la violence. L'un des risques majeurs encourus réside en la répétition de l'expérience vécue à partir de laquelle l'enfant pourra passer du statut de victime à celui d'agresseur, perpétuant ainsi les systèmes violents à travers les générations. Se 
pose ici la question délicate de la reproduction transgénérationnelle. Zaouche Gaudron et Molinier (2012) soulignent, en effet, qu'un homme qui a été confronté à la violence conjugale de ses parents durant son enfance a trois fois plus de risque de devenir violent. Cependant, dans notre pays, et à ce jour, les enfants exposés ne sont que rarement pris en charge par les structures existantes et, contrairement aux enfants victimes de maltraitances, il n'existe aucun dossier permettant de les repérer et de les aider à surmonter un tel traumatisme.

Notre étude, pourrait se poursuivre, avec pour objectif de mieux identifier les facteurs de risques et les facteurs de protection. Le protocole utilisé pourrait être poursuivi dans une démarche clinique afin que les professionnels puissent aménager des prises en charge adaptées à chaque situation rencontrée.

C'est dans une perspective de prévention qu'il est nécessaire d'accompagner et de soutenir ces enfants exposés, afin de « restaurer leurs capacités de penser, exactement comme la reprise d'un maillage du filet évitant l'effondrement psychique par la béance du traumatisme » (Vouche, 2011, 111). Nous considérons que l'enjeu consiste à repérer cette violence le plus précocement possible afin de mettre en œuvre une prise en charge spécifique adaptée à chaque enfant et limiter les effets délétères de l'exposition à la violence conjugale.

\section{Points à retenir :}

En nous basant sur le point de vue des enfants exposés à la violence conjugale, cette recherche propose d'analyser les représentations d'attachement auprès d'un échantillon clinique constitué de 10 enfants âgés de 3 à 7 ans. Pour ce faire, nous avons adapté le protocole de l'A.S.C.T (Bretherton, Ridgeway \& Cassidy, 1990). Le protocole utilisé pourrait être poursuivi dans une démarche clinique afin que les professionnels puissent envisager des prises en charge adaptées à chaque situation rencontrée et limiter les effets délétères de l'exposition à la violence conjugale. 
1. Bowlby J. (1973) Attachment and loss, Vol. 2: Separation: Anxiety and Anger. Hogard Press and the institute of Psycho-Analysis, London.

2. Bretherton I., Ridgeway D., Cassidy J. (1990). « Assessing internal working models of the attachment Relationship : An Attachment Story Completion Task for 3-yearsolds », in Greenberg M.T., Cischette D., Cummings E.M. (Eds.) : Attachment in the preschool years, University of Chicago Press, Chicago ; pp.3-49.

3. Commission Européenne (2006). «Vers une stratégie européenne sur les droits de l'enfant», Communication du 4 juillet 2006 (COM 2006/367); récupéré de http://eurlex.europa.eu/LexUriServ/LexUriServ.do?uri=COM:2006:0367:FIN:FR:HT

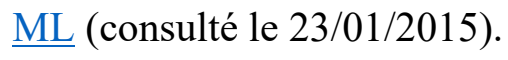

4. Macfie J., Fitzpatrick K.L., Rivas E.M., Cox M.J. (2008). «Independent influences upon mother-toddler role reversal: infant-mother attachment disorganization and role reversal in mother's childhood », Attachment Human Development ; 10 : 30-39.

5. Fortin A., Côté I., Rousseau S., Dubé M. (2007). Soutenir les mères pour prévenir les effets néfastes de la violence conjugale chez les enfants. Guide pour les intervenantes des maisons d'aide et d'hébergement. Centre de recherche interdisciplinaire sur la violence familiale et la violence faite aux femmes (CRI-VIFF), Montréal.

6. Fortin A., Lachance L. (2011). « La parentification chez l'enfant exposé à la violence conjugale », La Revue Internationale de l'Education Familiale ; 29 : 63-86.

7. Guédeney N. (2010) : L'attachement, un lien vital. Fabert, Paris.

8. Guédeney N., Guédeney A., Rabouam C. (2013). «Violences conjugales et attachement des jeunes enfants. Une revue de la littérature », Perspectives Psy ; 52 (3) : 222-230. 
9. IJzendoorn M.H., Bakermans-Kranenburg M.J. (2010). «Attachement sécurisé et désorganisé dans les familles et les orphelinats où il y a maltraitance », Encyclopédie sur le développement des jeunes enfants (sur Internet), Centre for Child and Family Studies, Leiden University, Pays Bas; récupéré de http://www.enfantencyclopedie.com/sites/default/files/textes-experts/fr/45/attachement-securise-et-

desorganise-dans-les-familles-et-les-orphelinats-ou-il-y-a-maltraitance.pdf (consulté le 24/01/2015).

10. Ijzendoorn M.H., Schuengel C., Bakermans-Kranenburg M. (1999). « Disorganized attachment in early childhood : a meta-analysis of precursors, concomitants and sequelae », Development and Psychopathology ; $11: 225-249$.

11. INED (2014). « Enquête nationale sur les violences subies et les rapports de genre (VIRAGE) ».; récupéré de https://www.ined.fr/fr/tout-savoir-population/memosdemo/focus/enquete-virage/ (consulté le 13/12/2014).

12. INSEE-ONDRP (2012). Les résultats des enquêtes «Cadre de vie et sécurité » $\begin{array}{lllll}\text { ONDRP } & 2007 & \text { à } & 2012 ; & \text { récupéré }\end{array}$ http://www.inhesj.fr/sites/default/files/rapport_ondrp_2012_3.pdf (consulté le $13 / 12 / 2014)$

13. Jaspard M., Brown E., Condon S., Fougeyrollas-Schwebel D., Houel A., Lhomond B., Maillochon F., Saurel-Cubizolles M.J., Schiltz M.A. (2003). Les violences envers les femmes en France. Une enquête nationale, La Documentation Française, Paris.

14. Lyons-Ruth K. (2007). «The interface between attachment and intersubjectivity: Perspective from the longitudinal study of disorganized attachment», Psychoanalytic Inquiry ; 26 (4) : 595-616. 
15. Lyons-Ruth K., Jacobvitz D. (1999). « Attachment disorganization : unresolved loss, relational violence and lapses in behavioral and attentional strategies », in Cassidy J., Shaver P. (Eds.) : Handbook of Attachment: Theory, Research and Clinical Application; p. 520-554, Guilford Press, New York.

16. Miljkovitch R., Pierrehumbert B. (2008). «Des stratégies comportementales d'attachement aux stratégies représentationnelles : construction et validité des cartes de codage pour les histoires à compléter », Enfance ; 60 (1) : 22-30.

17. Miljkovitch R., Pierrehumbert B., Karmaniola A., Halfon O. (2003). « Les représentations d'attachement du jeune enfant. Développement d'un système de codage pour les histoires à compléter », Devenir ; 15 (2) : 143-177.

18. Ministère des Droits des Femmes (2014). «Violences faites aux femmes »; récupéré de

http://femmes.gouv.fr/wpcontent/uploads/2014/03/Egalite_Femmes_Hommes_T6_bd. pdf (consulté le 12/12/2014).

19. ONED (2009). «L'enfant face à la violence dans le couple ». Actes de la journée du 4 décembre 2009; récupéré de http://www.oned.gouv.fr/system/files/publication/actes_enfantfacealaviolencedansleco uple_20091204_5.pdf (consulté le 13/12/2014).

20. Paul O., Zaouche Gaudron C. (2013). «L'adattamento dei bambini esposti alla violenza coniugale: l'approccio della sicurezza emotiva », Psicologia di comunità ; 9 : 23-31. DOI : 10.3280/PSC2013-002003.

21. Prior V., Glaser D. (2010). Comprendre l'attachement et les troubles de l'attachement : théorie, preuve et pratique. De Boeck., Bruxelles. 
22. Sadlier K. (Eds.) (2010). L'enfant face à la violence dans le couple. Dunod, Paris.

23. Savard N. (2011). Développement socio-affectif de l'enfant âgé de 5 à 6 ans en contexte de violence conjugale : une approche écosystémique. Thèse de Doctorat en Psychologie du Développement, Université Toulouse II, Toulouse.

24. Savard N., Zaouche Gaudron C. (2010). «Etat des lieux des recherches sur les enfants exposés à la violence conjugale », Neuropsychiatrie de l'Enfance et de l'Adolescence ; $58: 513-522$.

25. Savard N., Zaouche Gaudron C. (2011). «Points de repères pour examiner le développement de l'enfant exposé aux violences conjugales », La Revue Internationale de l'Education Familiale ; 29 : 13-35.

26. Savard N., Zaouche Gaudron C. (2013). «Recensement des actions évaluées à destination des enfants exposés à la violence conjugale », Psychologie Française ; 58 : 319-336.

27. Vouche J.P. (2011). «Les enfants exposés aux violences conjugales », in Coutanceau R., Smith J. (Eds.) : Violence et famille. Comprendre pour prévenir, Dunod, Paris ; p. 94-111.

28. Welzer-Lang D. (2005). Les hommes violents. Payot et rivages, Paris.

29. Zaouche Gaudron C., Molinier P. (2012). «Le ring conjugal : le chaos de la violence », in AÏN J. (Eds.) : Violences chaudes, violences froides. Érès, Toulouse ; p. 115-127.

30. Zaouche Gaudron C., Paul O. (2014). «Le développement socio-affectif des enfants exposés à la violence conjugale et leurs représentations de cette violence: Une approche de la sécurité émotionnelle », Rapport final de recherche. ONED, Paris. 
31. Zaouche Gaudron C., Pierrehumbert B. (2008). «Introduction au numéro thématique de Enfance : Comportement et représentation d'attachement dans l'enfance », Enfance $; 1: 5-10$.

32. Zeanah C., Danis B., Hirshberg L., Benoit D., Miller D., Heller S. (1999). « Disorganized attachment associated with Partner violence : A research note », Infant Mental Health Journal ; 20 (1) : 77-86. 
Tableau 1. Grille d'observation.

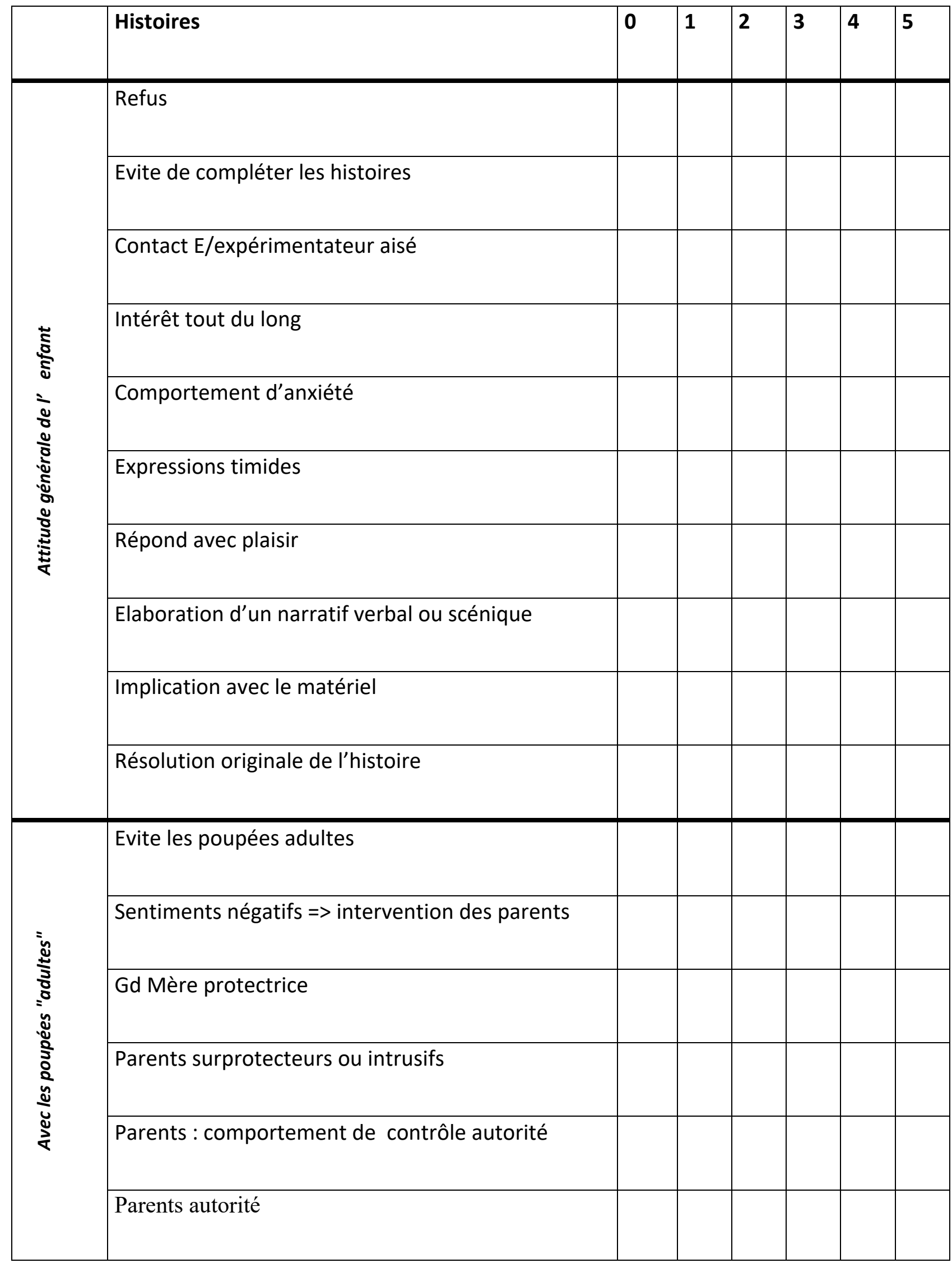




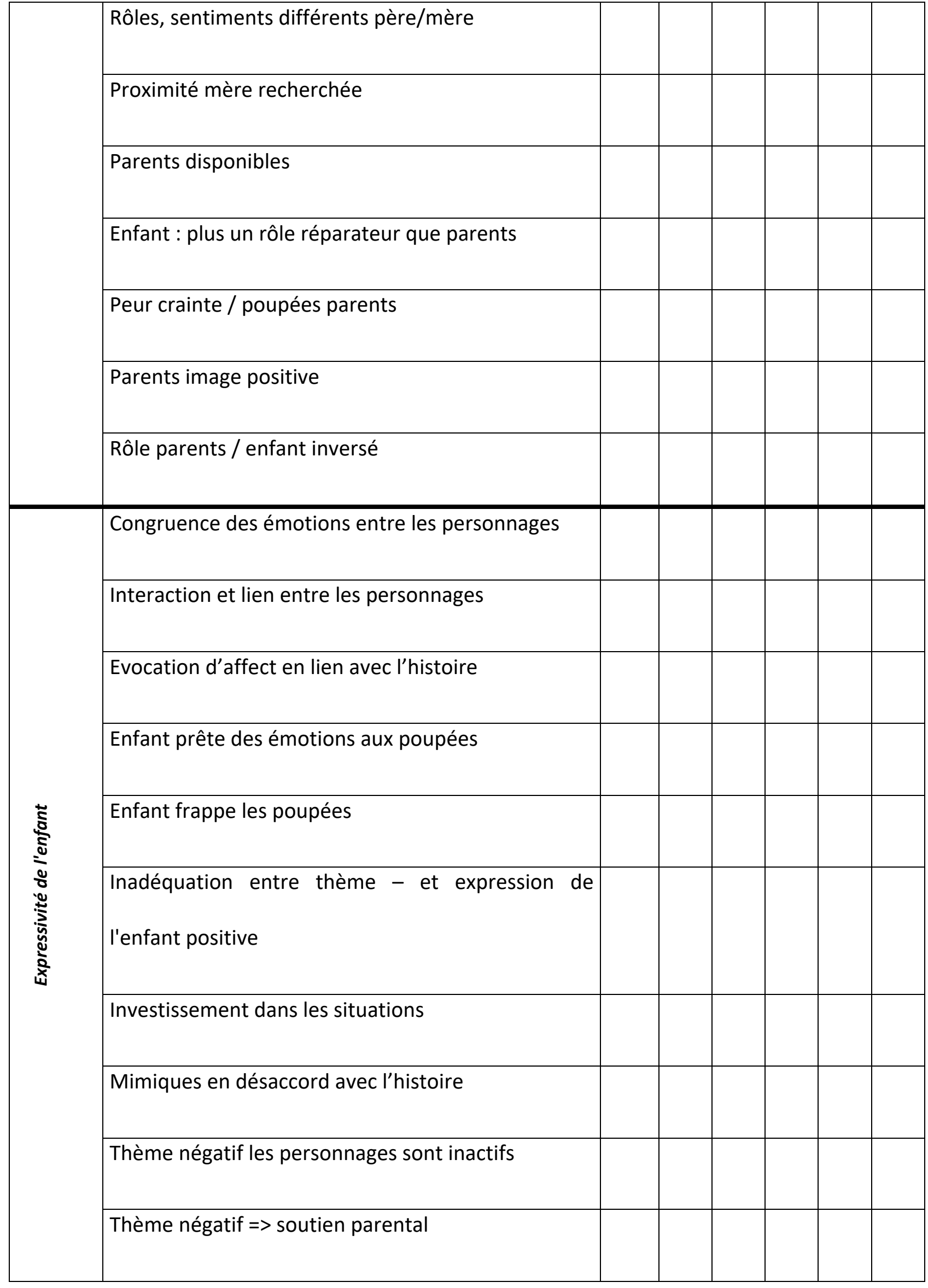




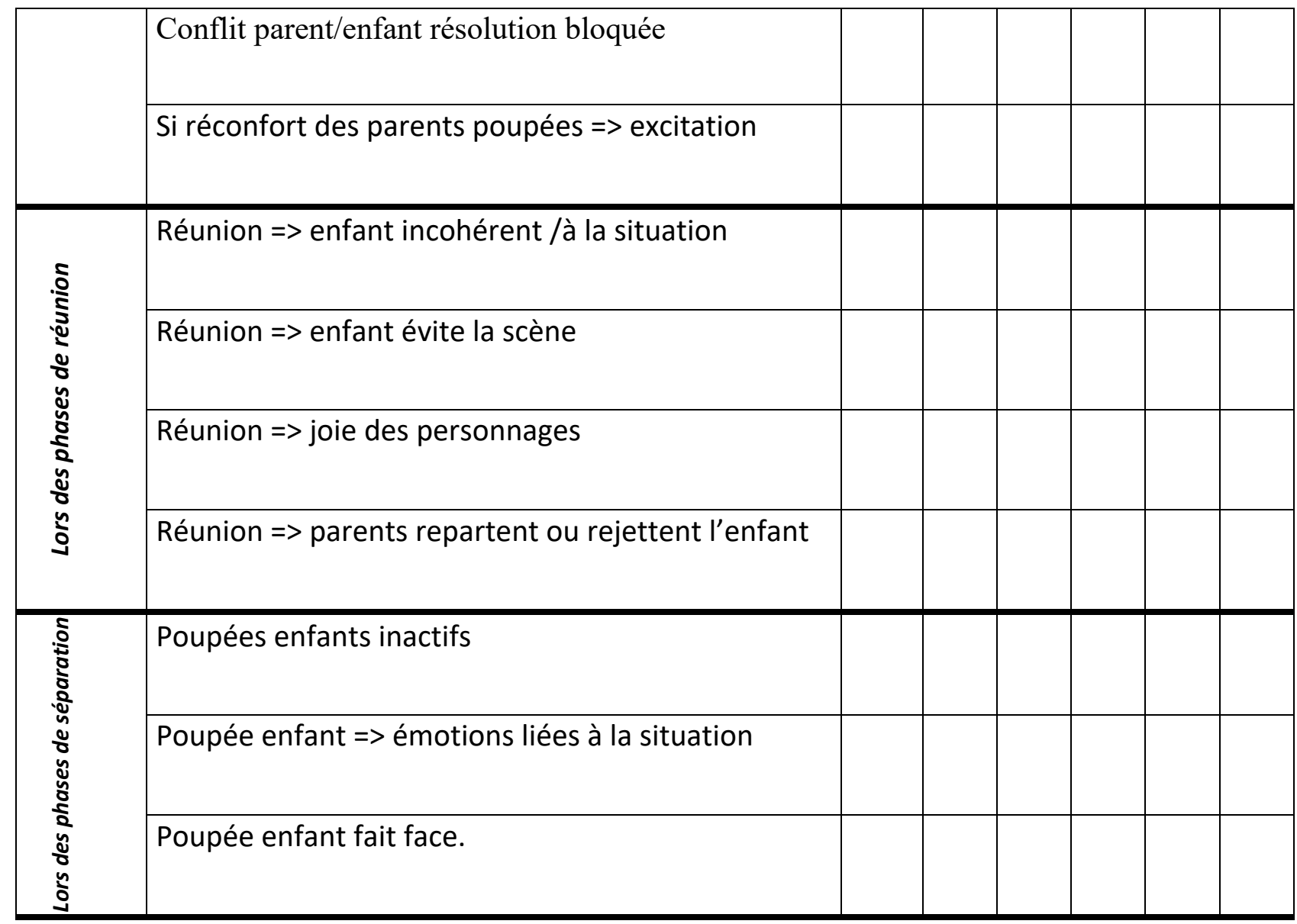


Tableau 2. Qualification de la qualité des représentations d'attachement de l'enfant

\begin{tabular}{|l|l|l|l|l|}
\hline & Sécurisé & Hyperactivé & Désactivé & Désorganisé \\
\hline Sécurisé & + de 45 & Peu importe & Peu importe & - de 65 \\
\hline Hyperactivé & - de 45 & $>$ désactivé & $<$ hyperactivé & - de 65 \\
\hline Désactivé & - de 45 & $<$ désactivé & $>$ hypercativé & - de 65 \\
\hline Désorganisé & Peu importe & Peu importe & Peu importe & + de 65 \\
\hline
\end{tabular}

Tableau 3. Répartition des scores T aux 4 dimensions d'attachement

\begin{tabular}{|l|r|r|r|r|r|l|}
\hline & Ages & Sécure & Désactivé & Hyperactivé & Désorganisé & Résultats \\
\hline Enfant 1 & 4 ans & 39,95 & 56,87 & 47,53 & 68,21 & Désorganisé \\
\hline Enfant 2 & 5 ans & 34,79 & 62,47 & 51,75 & 71,21 & Désorganisé \\
\hline Enfant 3 & 7 ans & 28,27 & 65,01 & 52,15 & 80,06 & Désorganisé \\
\hline Enfant 4 & 3 ans & 17,26 & 80,48 & 52,92 & 69,3 & Désorganisé \\
\hline Enfant 5 & 4 ans & 20,56 & 76,21 & 40,22 & 63,6 & Désactivé \\
\hline Enfant 6 & 6 ans & 27,61 & 73,76 & 42,91 & 60,96 & Désactivé \\
\hline Enfant 7 & 7 ans & 44,81 & 50,75 & 40,97 & 64,48 & Désactivé \\
\hline Enfant 8 & 5 ans & 18,14 & 76,82 & 58,69 & 76,54 & Désorganisé \\
\hline Enfant 9 & 7 ans & 37,11 & 57,07 & 44,52 & 63,59 & Désactivé \\
\hline Enfant 10 & 4 ans & 28,72 & 64,81 & 48,69 & 75,45 & Désorganisé \\
\hline
\end{tabular}

